\title{
Conservation of minor fruit genetic resources at the Botanical Garden, Bangladesh Agricultural University
}

\author{
M. Ashrafuzzaman, Most. Morsada Khatun, Noshin A. Tunazzina and \\ A.K.M. Golam Sarwar* \\ Laboratory of Plant Systematics, Department of Crop Botany, \\ Bangladesh Agricultural University, Mymensingh 2202, Bangladesh \\ *Email:drsarwar@bau.edu.bd \\ Received : 16.04.2021 ; Revised : 04.05.2021 ; Accepted : 06.05.2021
}

\begin{abstract}
Minor fruits (MFs), a good source of micronutrients, can contribute significantly to the nutritional requirement of the rural population and be an alternative source to combat hidden hunger caused by micronutrient deficiencies mainly vitamins and minerals. A detailed survey was conducted to update the checklist of the MF collections at Botanical Garden under the Department of Crop Botany, Bangladesh Agricultural University (BAUBG) and their conservation priority in national/international perspectives. A total of $108 \mathrm{MF}$ species, belong to 67 genera and 38 families, were collected and conserved at the BAUBG. Moraceae and Myrtaceae were the most dominant families with 8 taxa each followed by Rutaceae with 7 taxa, Arecaceae, Phyllanthaceae and Sapotaceae with 6 taxa each. Among the genera, Syzygium is the largest genus having 7 species followed by Citrus, Ficus and Garcinia with 5 species in each. Trees make up the largest proportion of MFs followed by shrubs, herbs and climbers. Although MFs are major sources of micronutrients, 97 taxa were also used in the treatment of various diseases in ethnobotanical literature. This study also contributes to the conservation database of plant genetic resources of BAUBG and the country as well.
\end{abstract}

Keywords: Botanical garden, conservation, medicinal uses, underutilized fruits

\section{INTRODUCTION}

Minor fruits (MFs) are a group of fruits presently growing in a scattered and unattended way on roadsides, homestead land, wasteland, etc. (Roya and Bauri, 2019). These fruits are cultivated to a limited extent only, and with consumption and trade being more limited both geographically and quantitatively, although many are of considerable economic importance in their respective regional markets. In general, these are suitable for human consumption but relatively less palatable than other major fruits (Srivastava et al., 2017); MF species act as life support species in extreme environmental conditions and threatened habitats and have the tolerance to survive under harsh climatic conditions. The MFs are sometimes the only source of protective food to meet the vitamins and minerals requirements, and integral part of traditional foods of the people living in (remote) villages and tribal areas (Krishna et al., 2019). These fruits play a great role in improving the food and nutritional status of the local people due to their year-round availability (Pasha and Uddin, 2019). The MFs, synonymously called underutilized fruits, remain underutilized because of underestimation of their potential uses, little known outside its growing area, nonavailability of their complete botanical information, inadequate research on their commercial exploitation, lack of knowledge on their food and nutritional value, promotion, popularization of very few fruit crops, fast disappearance of the ecosystem, and habitat destruction (Dandin and Kumar, 2016). Nowadays, for achieving the Sustainable Development Goals (SDGs), especially Goal \#2, which calls for the eradication of hunger and all forms of malnutrition; MFs have become the focus of attraction. Because they are found harbouring nutritionally rich compounds, climate-resilient, resistant to biotic \& abiotic stresses, rich in medicinal \& nutritive value, source of breeding materials for crop improvement (Ghosh, 2017; Krishna et al., 2019). The MFs are rich in phytochemicals and micronutrients such as antioxidants, polyphenols, flavonoids, minerals and vitamins, which are essential for good health and nutrition, advancing physical and intellectual development. Many of the MFs possess social and/ 
or ritual values and importance; these also provide the source of feed and nutrition for birds and wild animals.

Hitherto, a total of 255 minor edible fruit yielding species belonging to 149 genera under 61 families, three families to the Liliopsida and 58 families to Magnoliopsida, have been reported from the Bangladesh territory (Pasha and Uddin, 2019). Among these, 48 species are cultivated for fruits, 35 species both cultivated and wild, and the remaining species are exclusively wild. Due to rapid population growth and other anthropogenic activities, alien species, and climate change events - high temperature, change in rainfall pattern, early or late monsoon, frequent floods and cyclones, etc., these valuable plant genetic resources are now declining at an alarming rate. As a result, some of these valuable MF species are now become (critically) endangered and on the verge of extinction. Since its inception in 1963, the Botanical Garden under the Department of Crop Botany, Bangladesh Agricultural University (BAUBG) has been conducting collection and conservation activities. Presently about 1,146 species, more than $20 \%$ of the total Bangladesh (Spermatophyte) flora, under 327 genera and 215 families are harboured at this garden (Sarwar, 2019; 2020). Over time, an enormous number of MFs are also conserved in this place. Hitherto, five hundred twenty-seven medicinal and aromatic plant species, many of them are MFs, belonging to one hundred one families have conserved here (Sarwar, 2020). The objective of the present research was to compile an updated checklist of the MF collections of the BAUBG and their conservation priority from national/ international perspectives.

\section{METHODOLOGY}

A detailed survey on the MFs growing throughout BAUBG, located at $24^{\circ} 722463 \mathrm{~N}$ $90^{\circ} 442163 \mathrm{E}$, was carried out through frequent visits (Sarwar, 2020). During these visits, fresh flowering samples were collected; herbarium specimens were prepared as vouchers by drying the fresh samples properly. The dried specimens were mounted on the herbarium sheet and preserved in Prof. Dr Arshad Ali Herbarium at the Botanical Garden, Department of Crop Botany, Bangladesh Agricultural University. The collected fresh (or dried) specimens were identified in the field or by comparing with herbarium specimens or published literature. The botanical names were updated following <http://www.worldfloraonline.org> (older http://www.theplantlist.org) and their conservation status (and uses) follows "Encyclopedia of Flora and Fauna of Bangladesh" (Ahmed et al., 2008a, b, 2009a, b, c, d; Siddiqui et al., 2007) and "Red Data Book of Vascular Plants of Bangladesh" (Khan et al., 2001; Ara et al., 2013). The local names and medicinal uses were documented critically from published literature (Uddin, 2006; Yusuf et al., 2009; Uddin et al., 2016) and online resources.

\section{RESULTS AND DISCUSSION}

A total of 108 species were listed and distributed under 38 families and 67 genera (Table 1; Figs 16). Out of these, 30 species are exotic and mostly cultivated; however, a few of them, for example, Hibiscus sabdariffa, Opuntia dellenii, Pithecellobium dulce, Polyalthia suberosa, Tamarindus indica, etc., have become naturalized and found in the wild also (Pasha and Uddin, 2019). Moreover, Pasha and Uddin (2019) had identified 44 MFs species, many of them encompassed the BAUBG collection, as promising crops for the future due to their taste, colour, wide use and popularity in consumption. Moraceae and Myrtaceae were the most dominant families with 8 taxa each followed by Rutaceae with 7 taxa, Arecaceae, Phyllanthaceae and Sapotaceae with 6 taxa each, and Annonaceae with 5 taxa. The remaining families are represented by 4 or fewer species each (Table 2). Among the genera, Syzygium is the largest genus having 7 species $(6.48 \%$ of the total MFs). Citrus, Ficus and Garcinia come next with 5 species in each genus (4.63\%) followed by Annona, Antidesma, Ardisia, Diospyros, Elaeocarpus and Flacourtia with 3 species (2.78\%) in each genus (Table 3). These ten genera together account for more than one-third of the total number of MFs the BAUBG. The remaining 11 genera were documented by having 2 species each and 46 genera represented by only 1 species each (Table 1 ). Among these 108 species, tree species were dominant $(85 ; 78.70 \%)$ followed by shrubs $(15$; $13.89 \%)$, herbs $(5 ; 4.63 \%)$ and only $3(2.78 \%)$ climber (Fig. 7). The number of recorded MFs (255 
Table 1: Minor fruit plant species conserved at the Botanical Garden, Bangladesh Agricultural University. (Ex) Exotic; Clim. Climber; LC Least concern; NE Not evaluated; VU Vulnerable; NT Near threatened; EN Endangered; DD Data deficient; CD Conservation dependent

\begin{tabular}{|c|c|c|c|c|c|c|}
\hline $\begin{array}{l}\text { Sl. } \\
\text { No. }\end{array}$ & $\begin{array}{l}\text { Local } \\
\text { Name }\end{array}$ & Botanical Name & Family & Habit & Status & $\begin{array}{l}\text { Fig. } \\
\text { No. }\end{array}$ \\
\hline 1. & Bael & Aegle marmelos (L.) Corr. & Rutaceae & Tree & $\mathrm{LC}$ & $1 \mathrm{~A}$ \\
\hline 2. & Kaju badam & Anacardium occidentale L. (Ex) & Anacardiaceae & Tree & $\mathrm{LC}$ & $1 \mathrm{~B}$ \\
\hline 3. & Pond apple & Annona glabra L. (Ex) & Annonaceae & Tree & $\mathrm{NE}$ & $1 \mathrm{C}$ \\
\hline 4. & Nona-ata & Annona reticulata L. (Ex) & Annonaceae & Tree & $\mathrm{LC}$ & $1 \mathrm{D}$ \\
\hline 5. & Sharifa & Annona squamosa L. (Ex) & Annonaceae & Tree & $\mathrm{LC}$ & $1 \mathrm{E}$ \\
\hline 6. & Elena/Bignay & Antidesma acidum Retz. & Phyllanthaceae & Tree & $\mathrm{LC}$ & $1 \mathrm{~F}$ \\
\hline 7. & Choto Sialbuka & Antidesma bunius (L.) Spreng. & Phyllanthaceae & Tree & $\mathrm{LC}$ & $1 \mathrm{G}$ \\
\hline 8. & Siyal Buka & Antidesma montanum Blume & Phyllanthaceae & Tree & $\mathrm{LC}$ & - \\
\hline 9. & Chauldhoa & Ardisia humilis Vahl & Primulaceae & Shrub & $\mathrm{LC}$ & $1 \mathrm{H}$ \\
\hline 10. & Bonjami & Ardisia sanguinolenta Blume & Primulaceae & Shrub & $\mathrm{LC}$ & $1 \mathrm{I}$ \\
\hline 11. & Bonjam & Ardisia solanacea (Poir.) Roxb. & Primulaceae & Shrub & $\mathrm{LC}$ & $1 \mathrm{~J}$ \\
\hline 12. & Supari & Areca catechu L. & Arecaceae & Tree & $\mathrm{LC}$ & $1 \mathrm{~L}$ \\
\hline 13. & Dewa & Artocarpus lacucha Buch.-Ham. & Moraceae & Tree & $\mathrm{LC}$ & $1 \mathrm{M}$ \\
\hline 14. & Chapalish & Artocarpus chama Buch.-Ham. & Moraceae & Tree & $\mathrm{NE}$ & $1 \mathrm{~K}$ \\
\hline 15. & Bilimbi & Averrhoa bilimbi L. (Ex) & Oxalidaceae & Tree & $\mathrm{LC}$ & $1 \mathrm{~N}$ \\
\hline 16. & Kamranga & Averrhoa carambola L. (Ex) & Oxalidaceae & Tree & $\mathrm{LC}$ & 10 \\
\hline 17. & Latkan & Baccauria ramiflora Lour. & Phyllanthaceae & Tree & $\mathrm{LC}$ & $2 \mathrm{~A}$ \\
\hline 18. & Tal & Borassus flabellifer L & Arecaceae & Tree & $\mathrm{LC}$ & $2 B$ \\
\hline 19. & Betphal & Calamus manillensis L. & Arecaceae & Tree & - & $2 \mathrm{C}$ \\
\hline 20. & Kumbhi & Careya arborea Roxb. & Lecythidaceae & Tree & VU & $2 \mathrm{D}$ \\
\hline 21. & Karamcha & Carissa carandas L. (Ex) & Apocynaceae & Shrub & $\mathrm{LC}$ & $2 \mathrm{E}$ \\
\hline 22. & Bon supari & Caryota urens L. & Arecaceae & Tree & $\mathrm{LC}$ & $2 \mathrm{~F}$ \\
\hline 23. & Khejur & Chamaerops humilis L. (Ex) & Arecaceae & Tree & $\mathrm{LC}$ & $5 \mathrm{C}$ \\
\hline 24. & Star Apple & Chrysophyllum roxburghii G. Don (Ex) & Sapotaceae & Tree & $\mathrm{LC}$ & - \\
\hline 25. & Ada jamir & Citrus assamensis R.M. Dutta \& Bhatt. & Rutaceae & Shrub & Rare & $2 \mathrm{G}$ \\
\hline 26. & Satkora & Citrus aurantium L. & Rutaceae & Tree & $\mathrm{LC}$ & - \\
\hline 27. & Rough Lemon & Citrus jambhiri Lush & Rutaceae & Tree & - & - \\
\hline 28. & Batabi Lebu & Citrus maxima (Burm.) Merr. & Rutaceae & Tree & $\mathrm{LC}$ & $2 \mathrm{H}$ \\
\hline 29. & Komla & Citrus reticulata Blanco & Rutaceae & Tree & $\mathrm{LC}$ & $2 \mathrm{I}$ \\
\hline 30. & Chalta & Dillenia indica L. & Dilleniaceae & Tree & $\mathrm{LC}$ & $2 \mathrm{~J}$ \\
\hline 31. & Bon Chalta & Dillenia pentagyna Roxb. & Dilleniaceae & Tree & $\mathrm{LC}$ & $2 \mathrm{~K}$ \\
\hline 32. & Ashphal & Dimocarpus longan Lour. & Sapindaceae & Tree & NT & $2 \mathrm{~L}$ \\
\hline 33. & Beelati Gab & Diospyros blancoi A. DC (Ex) & Ebenaceae & Tree & $\mathrm{LC}$ & $2 \mathrm{M}$ \\
\hline 34. & Deshi Gab & Diospyros malabarica (Desr.) Kostel & Ebenaceae & Tree & $\mathrm{LC}$ & $2 \mathrm{~N}$ \\
\hline 35. & Gulal/Katgula & Diospyros racemosa Roxb. & Ebenaceae & Tree & Rare & - \\
\hline 36. & Rudhrakha & Elaeocarpus angustifolius Blume & Elaeocarpaceae & Tree & EN & - \\
\hline 37. & Jalpai & Elaeocarpus floribundus Blume & Elaeocarpaceae & Tree & $\mathrm{LC}$ & 20 \\
\hline 38. & Mala & Elaeocarpus grandiflorus Sm. & Elaeocarpaceae & Tree & $\mathrm{LC}$ & $3 \mathrm{~A}$ \\
\hline 39. & Loquat & Eriobotrya japonica (Thunb.) Lindl. (Ex) & Rosaceae & Tree & VU & 3B \\
\hline 40. & Surinum cherry & Eugenia uniflora L. (Ex) & Myrtaceae & Shrub & - & $3 \mathrm{C}$ \\
\hline 41. & Makhna & Euryale ferox Salisb. & Nymphaeaceae & Herb & $\mathrm{NE}$ & $3 \mathrm{D}$ \\
\hline 42. & Hostikorni dumur & Ficus auriculata Lour. & Moraceae & Tree & $\mathrm{LC}$ & $3 \mathrm{E}$ \\
\hline 43. & Fapa-dumur & Ficus fistulosa Reinw. ex Blume & Moraceae & Tree & Rare & $3 F$ \\
\hline 44. & Kak dumur & Ficus hispida L.f. & Moraceae & Tree & LC & $3 G$ \\
\hline 45. & Jog Dumur & Ficus racemosa $\mathrm{L}$. & Moraceae & Tree & NE & $3 \mathrm{H}$ \\
\hline 46. & Sadimadi dumur & Ficus semicordata Buch.-Ham. ex Sm. & Moraceae & Tree & Rare & $3 \mathrm{I}$ \\
\hline
\end{tabular}


Table 1 Contd.

\begin{tabular}{|c|c|c|c|c|c|c|}
\hline $\begin{array}{l}\text { Sl. } \\
\text { No. }\end{array}$ & $\begin{array}{l}\text { Local } \\
\text { Name }\end{array}$ & Botanical Name & Family & Habit & Status & $\begin{array}{l}\text { Fig. } \\
\text { No. }\end{array}$ \\
\hline 47. & Baichi & Flacourtia indica (Burm. f.) Merr. & Salicaceae & Shrub & $\mathrm{LC}$ & $3 \mathrm{~J}$ \\
\hline 48. & Tomytomy & Flacourtia inermis Roxb. & Salicaceae & Shrub & $\mathrm{LC}$ & \\
\hline 49. & Paniala & Flacourtia jangomas (Lour.) Raeusch. & Salicaceae & Tree & $\mathrm{LC}$ & $3 \mathrm{~K}$ \\
\hline 50. & Kaufal & Garcinia cowa Roxb. ex DC. & Clusiaceae & Tree & $\mathrm{LC}$ & $3 \mathrm{~L}$ \\
\hline 51. & Mangosteen & Garcinia mangostana L. & Clusiaceae & Tree & - & \\
\hline 52. & Gutta-gam & Garcinia morella (Gaertn.) Desr. & Clusiaceae & Tree & LC & \\
\hline 53. & Thoikar & Garcinia pedunculata Roxb. ex Buch-Ham. & Clusiaceae & Tree & $\mathrm{LC}$ & $3 \mathrm{M}$ \\
\hline 54. & Dephall & Garcinia xanthochymus Hook.f. ex Anders. & Clusiaceae & Tree & $\mathrm{LC}$ & $3 N$ \\
\hline 55. & Phalsa & Grewia asiatica L. & Malvaceae & Tree & $\mathrm{LC}$ & 30 \\
\hline 56. & Raktagota & $\begin{array}{l}\text { Haematocarpus validus (Miers) } \\
\text { Bakh.f. ex Forman }\end{array}$ & Menispermaceae & Clim. & Very Rare & $4 \mathrm{~A}$ \\
\hline 57. & Lal mesta & Hibiscus sabdariffa L. (Ex) & Malvaceae & Shrub & NE & - \\
\hline 58. & Bonchalita & Leea asiatica (L.) Ridsdale & Leeaceae & Tree & Very Rare & - \\
\hline 59. & Chagal ladi & Lepisanthes senegalensis (Poir.) Leenh. & Sapindaceae & Shrub & $\mathrm{LC}$ & 50 \\
\hline 60. & Kathbel & Limonia acidissima $\mathrm{L}$. & Rutaceae & Tree & LC & $4 \mathrm{~B}$ \\
\hline 61. & Mahua & $\begin{array}{l}\text { Madhuca longifolia (Koenig ex L.) } \\
\text { MacBr. }\end{array}$ & Sapotaceae & Tree & NE & $3 C$ \\
\hline 62. & Maila-am & Mangifera longipes Griff. & Anacardiaceae & Tree & Rare & $4 \mathrm{D}$ \\
\hline 63. & Uri Aam & Mangifera sylvatica Roxb. & Anacardiaceae & Tree & VU & $4 \mathrm{E}$ \\
\hline 64. & Khirni & Manilkara hexandra (Roxb.) Dubard & Sapotaceae & Tree & Rare & - \\
\hline 65. & Sofeda & $\begin{array}{l}\text { Manilkara zapota (L.) P. van } \\
\text { Royen (Ex) }\end{array}$ & Sapotaceae & Tree & LC & $4 \mathrm{~F}$ \\
\hline 66. & Datranga & Melastoma malabathricum L. (Ex) & Melastomataceae & shrub & $\mathrm{LC}$ & $4 \mathrm{G}$ \\
\hline 67. & Mainakanta & Meyna spinosa Roxb. ex Link & Rubiaceae & Shrub & $\mathrm{LC}$ & $4 \mathrm{H}$ \\
\hline 68. & Bakul & Mimusops elengi L. & Sapotaceae & Tree & LC & $4 \mathrm{I}$ \\
\hline 69. & Sajna & Moringa oleifera L. & Moringaceae & Tree & LC & $4 \mathrm{~J}$ \\
\hline 70. & Tut & Morus alba L. (Ex) & Moraceae & Tree & $\mathrm{LC}$ & $4 \mathrm{~K}$ \\
\hline 71. & China cherry & Muntingia calabura L. & Muntingiaceae & Tree & - & $4 \mathrm{~L}$ \\
\hline 72. & Paddo, Komol & Nelumbo nucifera Gaertn. & Nelumbonaceae & Herb & $\mathrm{LC}$ & $4 \mathrm{M}$ \\
\hline 73. & Phanimanasa & Opuntia dellenii Haw. (Ex) & Cactaceae & shrub & $\mathrm{LC}$ & $4 \mathrm{~N}$ \\
\hline 74. & Passion fruit & Passiflora edulis Sims. (Ex) & Passifloraceae & Clim. & $\mathrm{LC}$ & 40 \\
\hline 75. & Jhumka lata & Passiflora foetida L. (Ex) & Passifloraceae & Clim. & $\mathrm{LC}$ & $5 \mathrm{~A}$ \\
\hline 76. & Avocado & Persea americana P. Mill. (Ex) & Lauraceae & Tree & CD & - \\
\hline 77. & Khudi Khejur & Phoenix acaulis Buch.-Ham. ex Roxb. & Arecaceae & Tree & VU & $5 B$ \\
\hline 78. & Orboroi & Phyllanthus acidus (L.) Skeels. & Phyllanthaceae & Tree & LC & $5 \mathrm{D}$ \\
\hline 79. & Amloki & Phyllanthus emblica L. & Phyllanthaceae & Tree & $\mathrm{LC}$ & $5 \mathrm{E}$ \\
\hline 80. & Khai babla & $\begin{array}{l}\text { Pithecellobium dulce (Roxb.) } \\
\text { Benth.(Ex) }\end{array}$ & Leguminosae & Tree & $\mathrm{LC}$ & - \\
\hline 81. & Murmuri & Polyalthia suberosa (Roxb.) Thw. (Ex) & Annonaceae & Tree & Rare & $5 \mathrm{~F}$ \\
\hline 82. & Mock strawberry & Potentilla indica (Jacks.) Th.Wolf & Rosaceae & Herb & LC & $5 G$ \\
\hline 83. & Gutgutya/Neur & Protium serratum (Wall. ex Coelbr.) Engl. & Burseraceae & Tree & $\mathrm{LC}$ & $5 \mathrm{H}$ \\
\hline 84. & Alu Bukhara & $\begin{array}{l}\text { Prunus bokhariensis Royle ex C.K. } \\
\text { Schneid. (Ex) }\end{array}$ & Rosaceae & Shrub & $\mathrm{LC}$ & $5 \mathrm{I}$ \\
\hline 85. & Buddha Narical & Pterygota alata (Roxb.) R.Br. & Malvaceae & Tree & LC & $5 \mathrm{~J}$ \\
\hline 86. & Dalim & Punica granatum L. (Ex) & Lythraceae & Tree & $\mathrm{LC}$ & $5 \mathrm{~K}$ \\
\hline 87. & $\begin{array}{l}\text { Sawtooth } \\
\text { blackberry }\end{array}$ & Rubus argutus Link. & Rosaceae & Tree & $\mathrm{LC}$ & - \\
\hline 88. & Santol fruit & $\begin{array}{l}\text { Sandoricum koetjape (Burm.f.) } \\
\text { Merr. (Ex) }\end{array}$ & Meliaceae & Tree & $\mathrm{LC}$ & $5 \mathrm{~L}$ \\
\hline 89. & Joyna & Schleichera oleosa (Lour.) Merr. & Sapindaceae & Tree & Rare & $5 \mathrm{M}$ \\
\hline
\end{tabular}


Ashrafuzzaman et al.

Table 1 Contd.

\begin{tabular}{|c|c|c|c|c|c|c|}
\hline & $\begin{array}{l}\text { Local } \\
\text { Name }\end{array}$ & Botanical Name & Family & Habit & Status & $\begin{array}{l}\text { Fig. } \\
\text { No. }\end{array}$ \\
\hline 90. & Choila & Sonneratia caseolaris (L.) Engl. & Lythraceae & Tree & NE & $5 \mathrm{~N}$ \\
\hline 91. & Amra & Spondias pinnata (L.f.) Kurz & Anacardiaceae & Tree & $\mathrm{LC}$ & $6 \mathrm{~A}$ \\
\hline 92. & Beelati Amra & Spondias purpurea L. (Ex) & Anacardiaceae & Tree & $\mathrm{LC}$ & - \\
\hline 93. & Miracle Fruit & $\begin{array}{l}\text { Synsepalum dulcificum (Schumach. } \\
\& \text { Thonn.) Daniell }\end{array}$ & Sapotaceae & Tree & Rare & $6 \mathrm{~B}$ \\
\hline 94. & Noli Jam & $\begin{array}{l}\text { Syzygium claviflorum(Roxb.) } \\
\text { Wall. ex A.M. Cowan \& Cowan }\end{array}$ & Myrtaceae & Tree & $\mathrm{LC}$ & $6 \mathrm{C}$ \\
\hline 95. & Kalo-Jam & Syzygium cuminii (L.) Skeels & Myrtaceae & Tree & $\mathrm{LC}$ & $6 \mathrm{D}$ \\
\hline 96. & Khudi jam & Syzygium cymosum (Lam.) DC. & Myrtaceae & Tree & $\mathrm{NE}$ & $6 \mathrm{E}$ \\
\hline 97. & Dhaki-jam & Syzygium grande (Wight) Walp. & Myrtaceae & Tree & $\mathrm{LC}$ & $6 \mathrm{~F}$ \\
\hline 98. & Golab-jam & Syzygium jambos (L.) Alston (Ex) & Myrtaceae & Tree & LC & 6G \\
\hline 99. & Malay apple & $\begin{array}{l}\text { Syzygium malaccense (L.) Merr. \& } \\
\text { Perry (Ex) }\end{array}$ & Myrtaceae & Tree & LC & $6 \mathrm{H}$ \\
\hline 100. & Jamrul & $\begin{array}{l}\text { Syzygium samarangense (Blume) } \\
\text { Merr. \& Perry (Ex) }\end{array}$ & Myrtaceae & Tree & $\mathrm{LC}$ & $6 \mathrm{I}$ \\
\hline 101. & Tentul & Tamarindus indica L. (Ex) & Leguminosae & Tree & $\mathrm{LC}$ & $6 \mathrm{~J}$ \\
\hline 102. & Bohera & Terminalia bellerica (Gaertn.) Roxb. & Combretaceae & Tree & LC & $6 \mathrm{~K}$ \\
\hline 103. & Horitoki & Terminalia chebula Retz. & Combretaceae & Tree & VU & $6 \mathrm{~L}$ \\
\hline 104. & Kantasingra & $\begin{array}{l}\text { Trapa natans var. bispinosa (Roxb.) } \\
\text { Makino }\end{array}$ & Lythraceae & Herb & $\mathrm{LC}$ & $6 \mathrm{M}$ \\
\hline 105. & Paniphal & Trapa natans L. & Lythraceae & Herb & LC & $6 \mathrm{~N}$ \\
\hline 106. & Bonlichu & $\begin{array}{l}\text { Xerospermum noronhianum (Blume) } \\
\text { Blume }\end{array}$ & Sapindaceae & Tree & Rare & - \\
\hline 107. & Jangli-kul & Zizyphus glabrata Heyne ex Roth & Rhamnaceae & Tree & EN & - \\
\hline 108. & Jangli Boroi & Zizyphus oenoplia (L.) Mill. & Rhamnaceae & Shrub & Rare & 60 \\
\hline
\end{tabular}

taxa) in Bangladesh territory is very smaller compared to the world record $(27,400)$ (French, 2019); therefore, a comprehensive field survey, exploitation, conservation and (large-scale) cultivation, through cultivar development, of MF genetic resources is strongly recommended. Many MF species, also known as "Food for the Poor" (Dandin and Kumar, 2016), are very nutritious, climate-resilient, well adapted to marginal lands and with low-cost inputs, thus may be of great benefit for the survival of poor communities and sustainability of agricultural ecosystems (Saúco, 2008; Krishna et al., 2019). The production (and trade) of MFs could play an important role not only in food and nutrition security but also as a source of income (Altendorf, 2018).

Although MFs are commonly used/consumed as sources of micronutrients and phytochemicals vitamins and minerals, antioxidants, etc., most of these (97 taxa) also have multiple (Ethno-) medicinal uses (Table 4). Along with the treatment of some common diseases e.g., cold, fever, cough, stomachache, asthma, scabies, skin diseases, etc., these MFs are also used for the treatment and/or lowering the risk of some of the deadliest diseases of the world e.g., cardiac problem, diarrhoea and dysentery, diabetes, respiratory tract infection, etc. (https://www.who.int/news-room/fact-sheets/ detail/the-top-10-causes-of-death). The underutilized MF crops have also vast potential for the production of value-added products, with high therapeutic, medicinal values and antioxidant properties, and free from the residue of toxic chemicals (Krishna et al., 2019). Among these species, some MFs e.g., amloki, bael, amrul, bilimbi, horitoki, bohera, kathbel, tentul, ber, kalojam/jamun, pomegranate, kamranga, etc., are very common and most popular among the rural people for their medicinal values. Many other fruit species are utilized by the tribal people. Eight MF species were recognized as threatened and/or nearthreatened viz. endangered 2, vulnerable 5 and nearthreatened 1, according to IUCN Red List categories $<$ https://www.iucnredlist.org/>; thirteen 

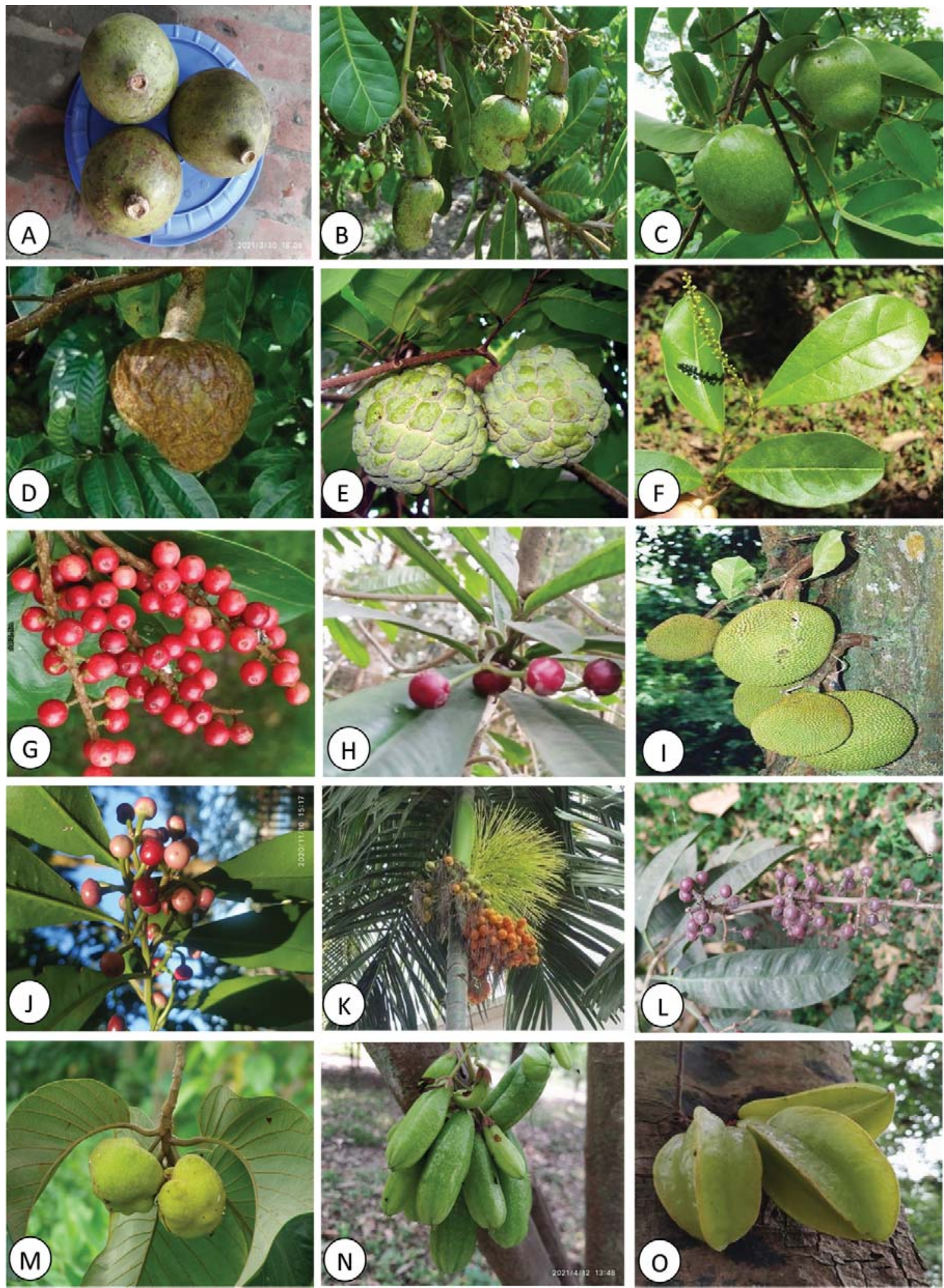

Figure 1: Photographs of minor fruit plants conserved at the Botanic Garden, Bangladesh Agricultural University. A. Aegle marmelos; B. Anacardium occidentale; C. Annona glabra; D. Annona reticulata; E. Annona squamosa; F. Antidesma acidum; G. Antidesma bunius; H. Ardisia humilis; I. Ardisia sanguinolenta; J. Ardisia solanacea; K. Areca catechu; L. Artocarpus chama; M. Artocarpus lacucha; N. Averrhoa bilimbi; O. Averrhoa carambola. 

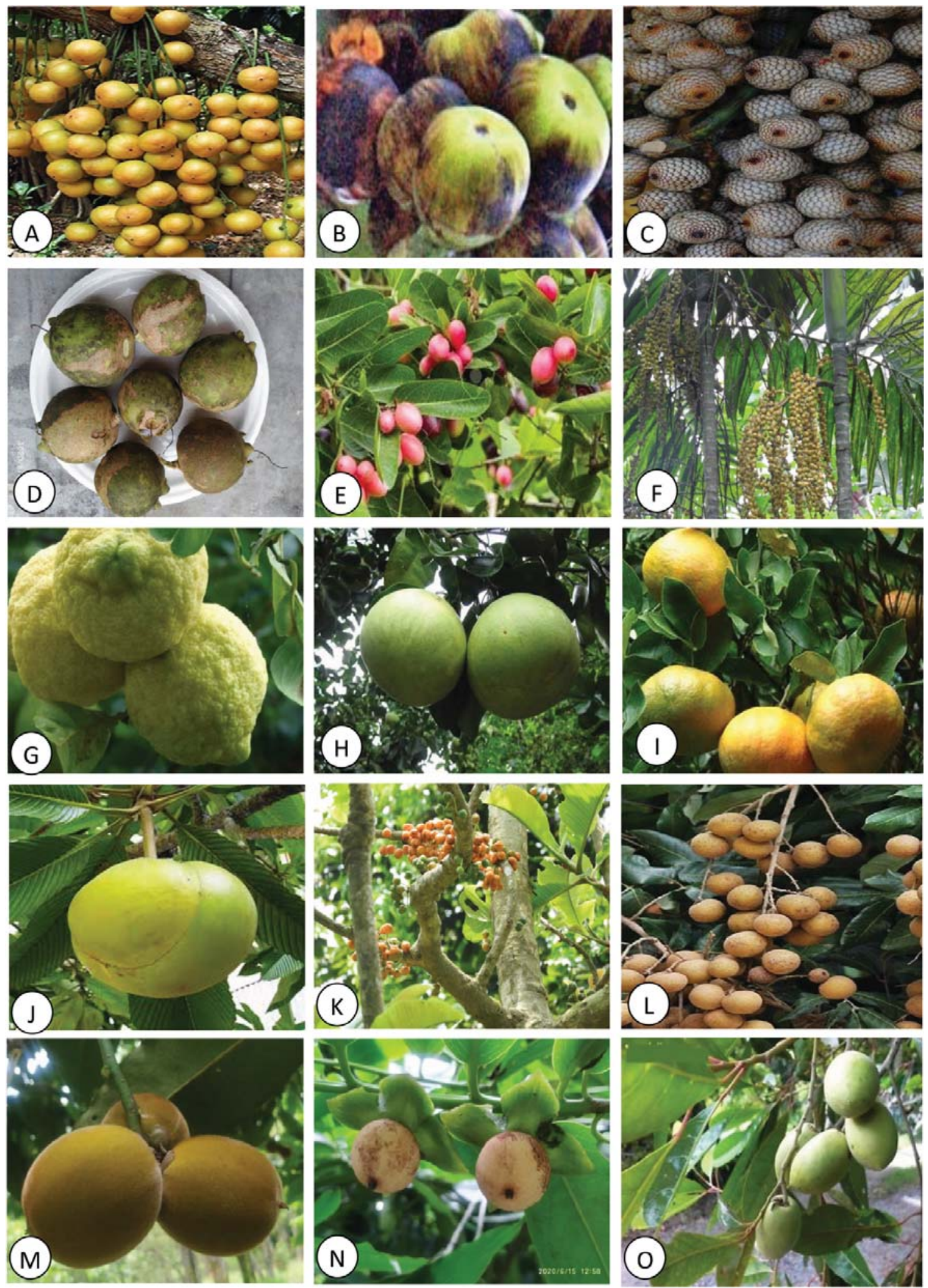

Figure 2: Photographs of minor fruit plants conserved at the Botanic Garden, Bangladesh Agricultural University.

A. Baccauria ramiflora; B. Borassus flabellifer; C. Calamus manillensis; D. Careya arborea; E. Carissaa carandas;

F. Caryota urens; G. Citrus assamensis; H. Citrus maxima; I. Citrus reticulata; J. Dillenia indica; K. Dillenia pentagyna; L. Dimocarpus longan; M. Diospyros blancoi; N. Diospyros malabarica; O. Elaeocarpus floribundus. 

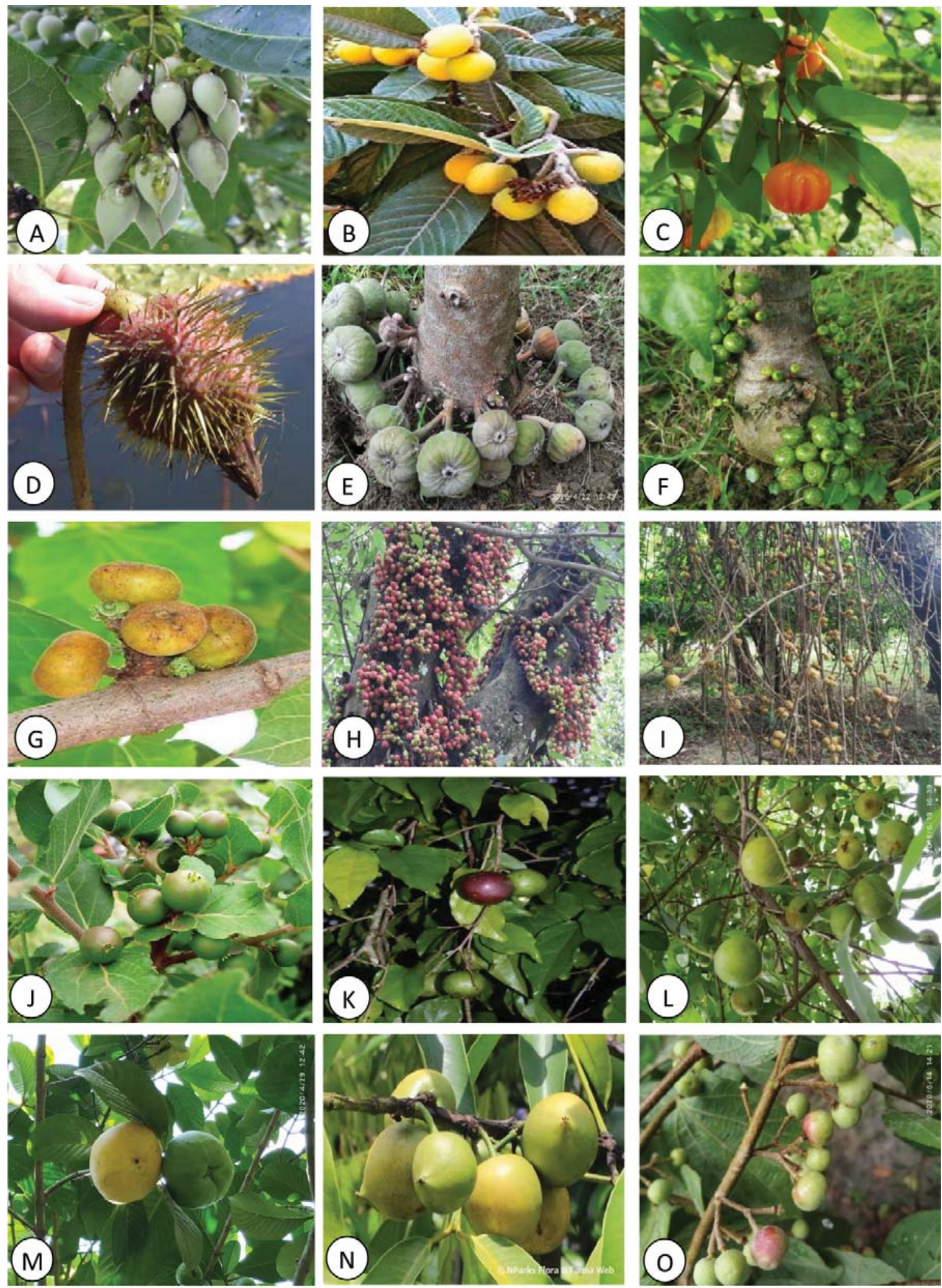

Figure 3: Photographs of minor fruit plants conserved at the Botanic Garden, Bangladesh Agricultural University. A. Elaeocarpus grandiflorus; B. Eriobotrya japonica; C. Eugenia uniflora; D. Euryale ferox; E. Ficus auriculata;

F. Ficus fistulosa; G. Ficus hispida; H. Ficus racemosa; I. Ficus semicordata; J. Flacourtia indica; K. Flacourtia jangomas; L. Garcinia cowa; M. Garcinia pedunculata; N. Garcinia xanthochymus; O. Grewia asiatica. 
Ashrafuzzaman et al.
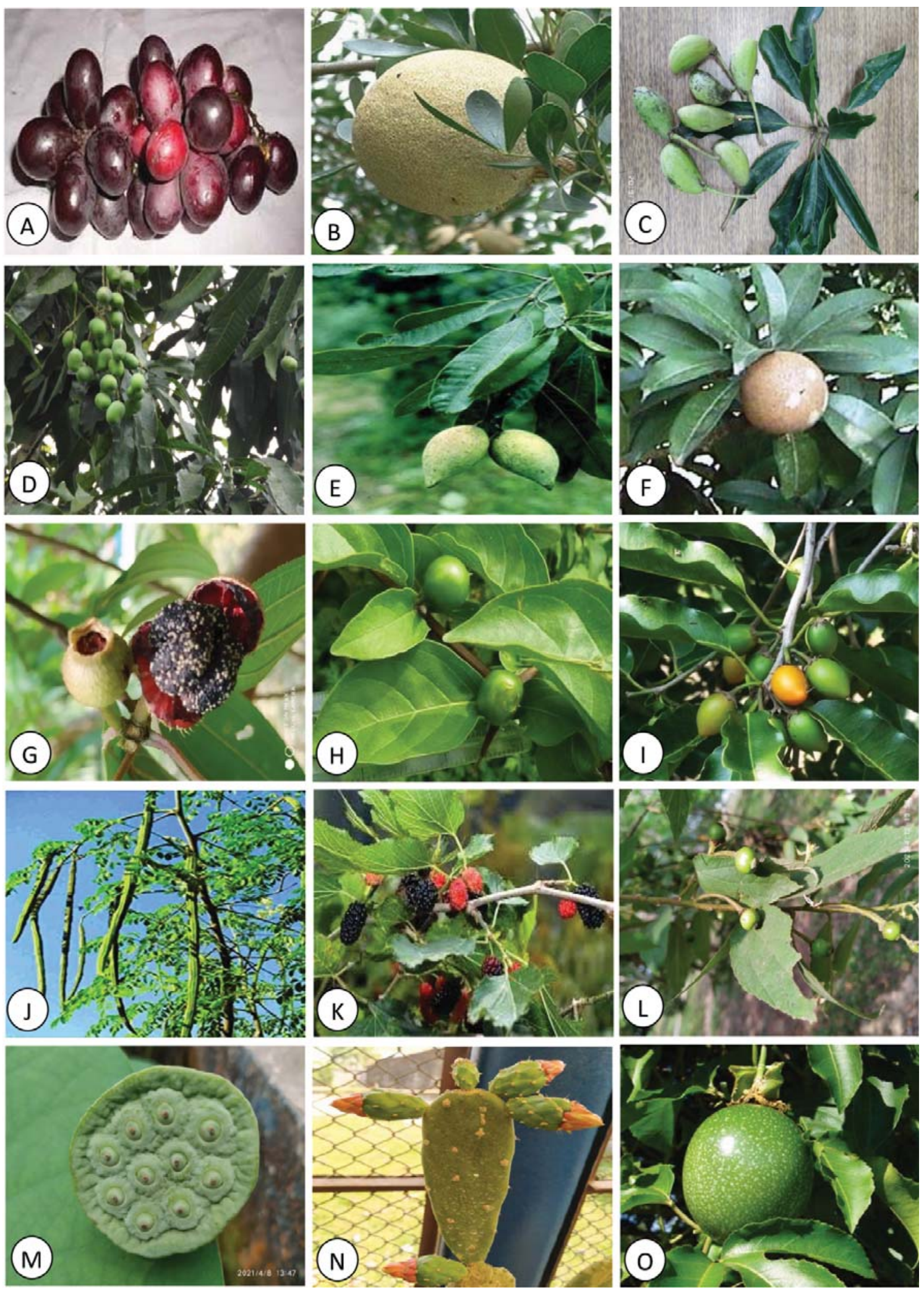

Figure 4: Photographs of minor fruit plants conserved at the Botanic Garden, Bangladesh Agricultural University.

A. Haematocarpus validus; B. Limonia acidissima; C. Madhuca longifolia; D. Mangifera longipes; E. Mangifera sylvatica; F. Manikara zapota; G. Melastoma malabathricum; H. Meyna spinosa; I. Mimusops elengi; J. Moringa oleifera; K. Morus alba; L. Muntingia calabura; M. Nelumbo nucifera; N. Opuntia dellenii; (O) Passiflora edulis. 

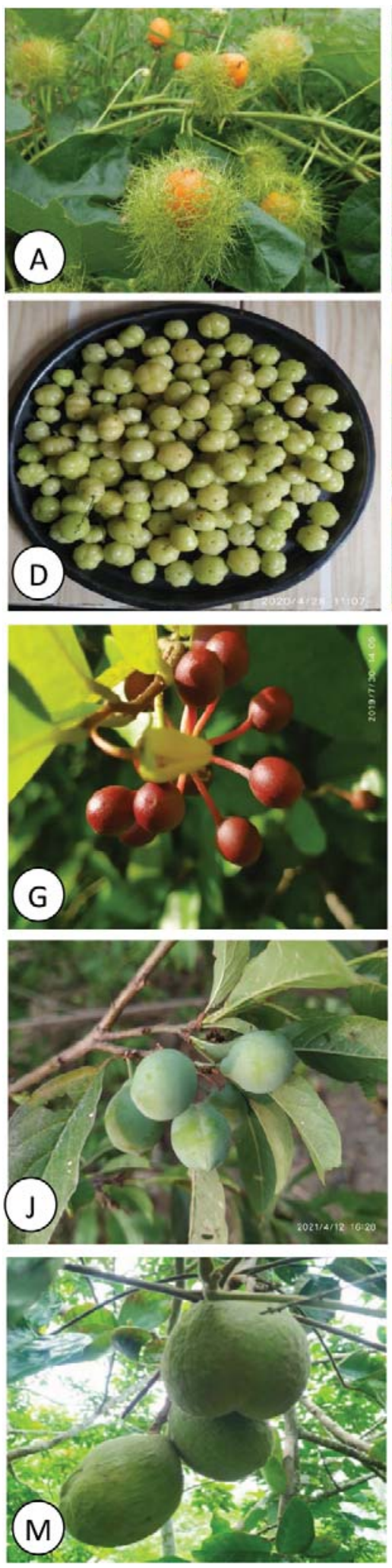
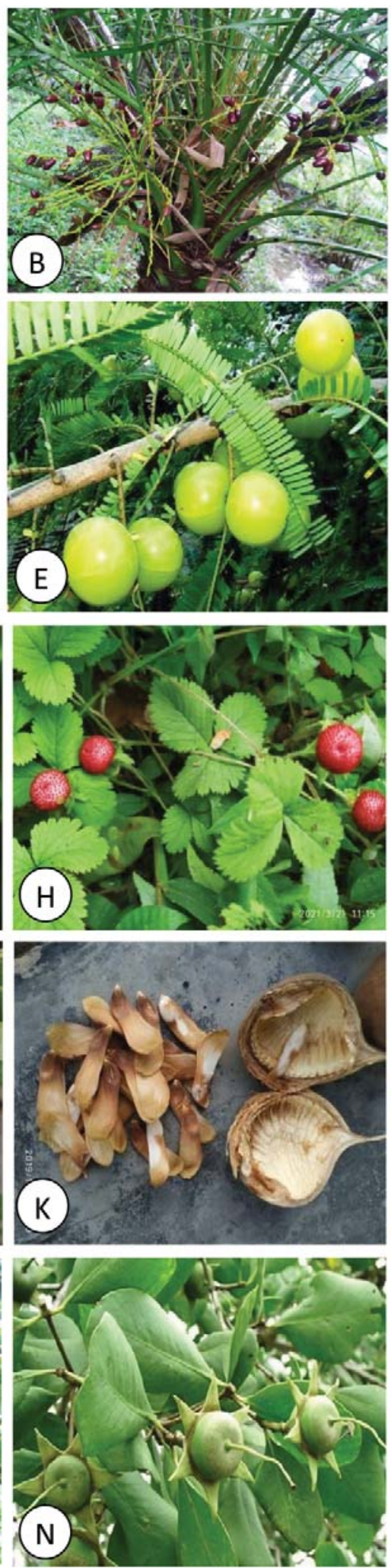
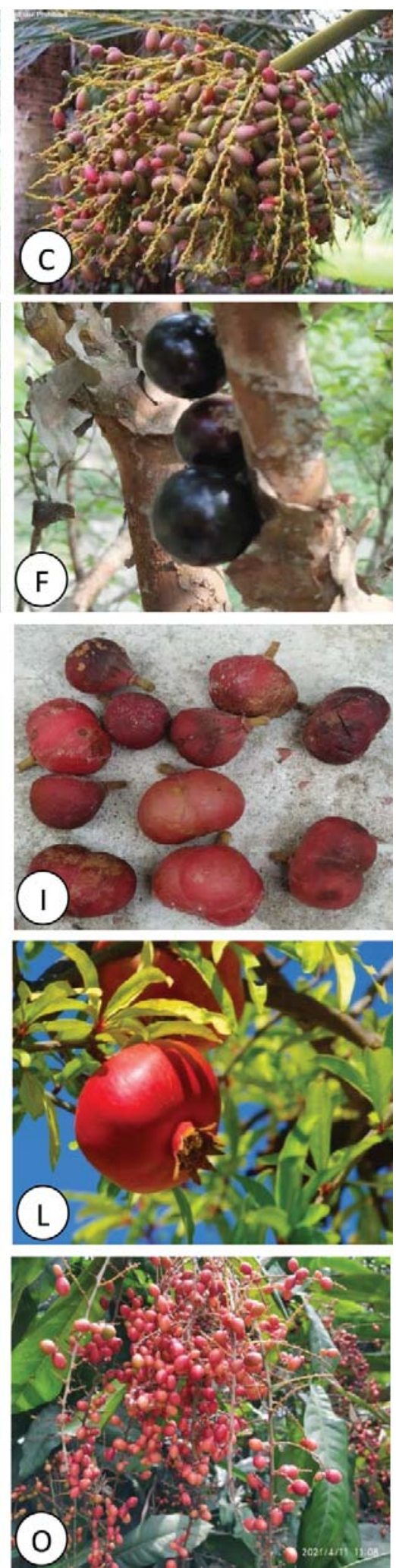

Figure 5: Photographs of minor fruit plants conserved at the Botanic Garden, Bangladesh Agricultural University. A. Passiflora foetida; B. Phoenix acaulis; C. Chamaerops humilis; D. Phyllanthus acidus; E. Phyllanthus emblica; F. Plinia cauliflora; G. Polyalthia suberosa; H. Potentilla indica; I. Protium serratum; J. Prunus bokhariensis; K. Pterygota alata; L. Punica granatum; M. Sandoricum koetjape; N. Sonneratia caseolaris; O. Lepisanthes senegalensis 

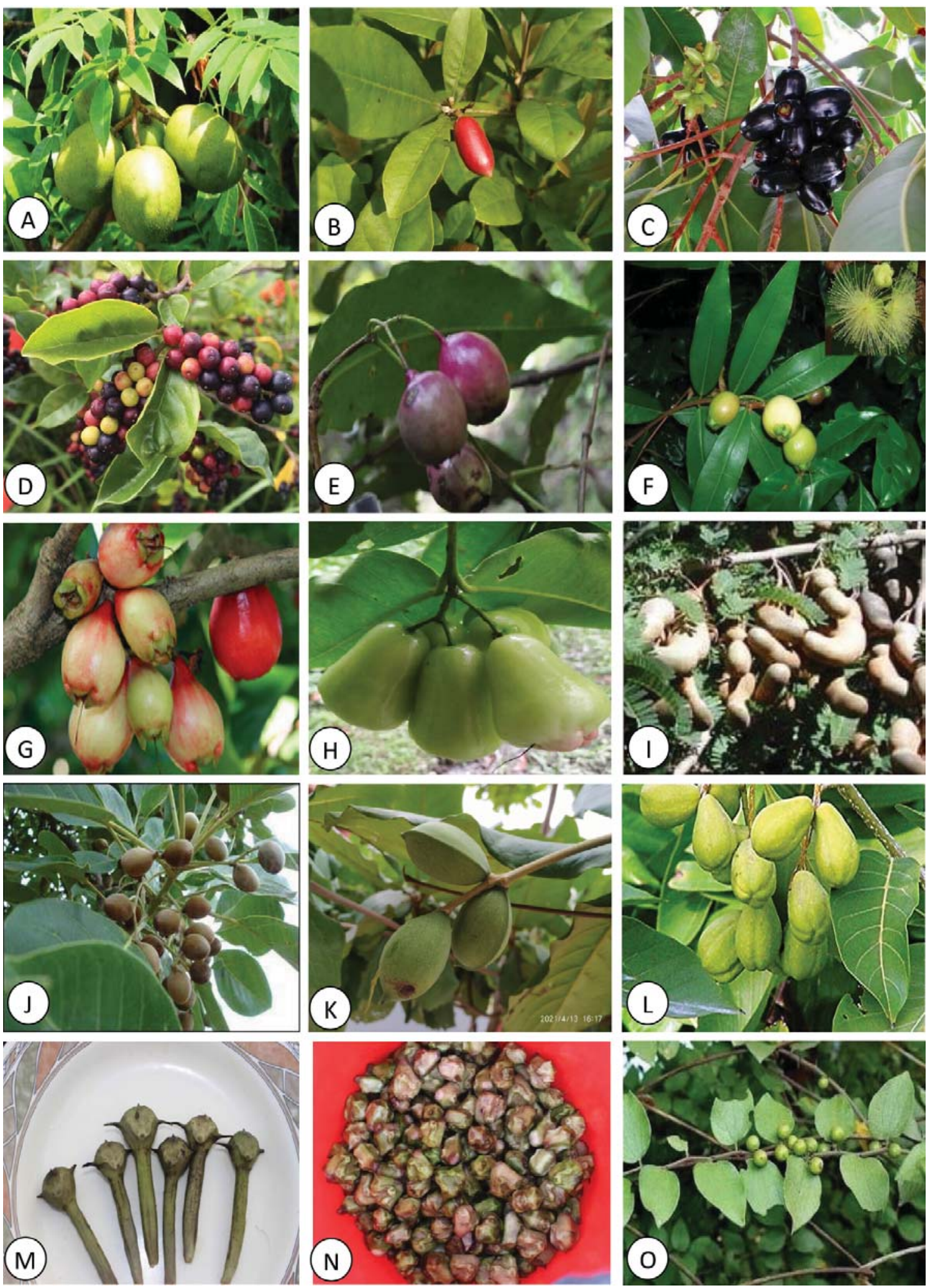

Figure 6: Photographs of minor fruit plants conserved at the Botanic Garden, Bangladesh Agricultural University. A. Spondias pinnata; B. Synsepalum dulcificum; C. Syzygium cuminii; D. Syzygium cymosum; E. Syzygium firmum; F. Syzygium jambos; G. Syzygium malaccense; H. Syzygium samarangense; I. Tamarindus indica; J. Terminalia bellerica; K. Terminalia catappa; L. Terminalia chebula; M. Trapa natans var. bispinosa; N. Trapa natans; O. Zizyphus oenoplia. 
Table 2: Family-wise distribution of minor fruit taxa at the Botanical Garden, Bangladesh Agricultural University

\begin{tabular}{|c|c|c|c|c|}
\hline Sl. No. & Family & Genera & Species & $\%$ of taxa in total \\
\hline 1 & Anacardiaceae & 3 & 5 & 4.63 \\
\hline 2 & Annonaceae & 2 & 4 & 3.70 \\
\hline 3 & Apocynaceae & 1 & 1 & 0.93 \\
\hline 4 & Arecaceae & 6 & 6 & 5.56 \\
\hline 5 & Burseraceae & 1 & 1 & 0.93 \\
\hline 6 & Cactaceae & 1 & 1 & 0.93 \\
\hline 7 & Clusiaceae & 1 & 5 & 4.63 \\
\hline 8 & Combretaceae & 1 & 2 & 1.85 \\
\hline 9 & Dilleniaceae & 1 & 2 & 1.85 \\
\hline 10 & Ebenaceae & 1 & 3 & 2.78 \\
\hline 11 & Elaeocarpaceae & 1 & 3 & 2.78 \\
\hline 12 & Lauraceae & 1 & 1 & 0.93 \\
\hline 13 & Lecythidaceae & 1 & 1 & 0.93 \\
\hline 14 & Leeaceae & 1 & 1 & 0.93 \\
\hline 15 & Leguminosae & 2 & 2 & 1.85 \\
\hline 16 & Lythraceae & 3 & 4 & 3.70 \\
\hline 17 & Malvaceae & 3 & 3 & 2.78 \\
\hline 18 & Melastomataceae & 1 & 1 & 0.93 \\
\hline 19 & Meliaceae & 1 & 1 & 0.93 \\
\hline 20 & Menispermaceae & 1 & 1 & 0.93 \\
\hline 21 & Moraceae & 3 & 8 & 7.40 \\
\hline 23 & Moringaceae & 1 & 1 & 0.93 \\
\hline 24 & Muntingiaceae & 1 & 1 & 0.93 \\
\hline 25 & Myrtaceae & 2 & 8 & 7.41 \\
\hline 26 & Nelumbonaceae & 1 & 1 & 0.93 \\
\hline 27 & Nymphaeaceae & 1 & 1 & 0.93 \\
\hline 28 & Oxalidaceae & 1 & 2 & 1.85 \\
\hline 29 & Passifloraceae & 1 & 2 & 1.85 \\
\hline 30 & Phyllanthaceae & 3 & 6 & 5.56 \\
\hline 31 & Primulaceae & 1 & 3 & 2.78 \\
\hline 32 & Rhamnaceae & 1 & 2 & 1.85 \\
\hline 33 & Rosaceae & 4 & 4 & 3.70 \\
\hline 34 & Rubiaceae & 1 & 1 & 0.93 \\
\hline 35 & Rutaceae & 3 & 7 & 6.48 \\
\hline 36 & Salicaceae & 1 & 3 & 2.78 \\
\hline 37 & Sapindaceae & 4 & 4 & 3.70 \\
\hline \multirow[t]{2}{*}{38} & Sapotaceae & 5 & 6 & 5.56 \\
\hline & Grand Total & 67 & 108 & 100 \\
\hline
\end{tabular}

species are rare/very rare in the wild. Another institute, the Germplasm Centre (of Fruit Tree Improvement Project), of Bangladesh Agricultural University is also maintaining the germplasms of 67 MFs of Bangladesh (Rahim et al., 2011). Only about one-third of MFs (of Bangladesh) were under cultivation (Pasha and Uddin, 2019), others are collected directly from the wild. The reckless collection and over-exploitation from the wild and illegal trade are some of the major drivers of biodiversity losses in the country (Sarwar, 2019). Awareness of the importance and utility of the 
Table 3: Top ten species-rich genera of minor

fruits at the Botanical Garden, Bangladesh Agricultural University

\begin{tabular}{lcc}
\hline Genus & No. of species & \% in total \\
\hline Syzygium & 7 & 6.48 \\
Citrus & 5 & 4.63 \\
Ficus & 5 & 4.63 \\
Garcinia & 5 & 4.63 \\
Annona & 3 & 2.78 \\
Antidesma & 3 & 2.78 \\
Ardisia & 3 & 2.78 \\
Diospyros & 3 & 2.78 \\
Elaeocarpus & 3 & 2.78 \\
Flacourtia & 3 & 2.78 \\
\hline
\end{tabular}

underutilized fruit species should be enhanced to encourage and engage the local people/ communities to participate in the conservation efforts. Recently, the Bangladesh Government enhanced the Protected Area (PA) management strategies by recognizing the benefits of collaboration with local communities in their management (DoE, 2015). The co-management, inclusion of local people/beneficiaries in management, of PAs coupled with alternative livelihood opportunities had given impacts biodiversity conservation. Moreover, most of the MF crops are perennial in nature, its cultivation has other benefits for example reduces environmental pollution, improves ecological balance, helps soil and water conservation and also enhances the beauty of the surroundings.

\section{CONCLUSION}

Out of the total $255 \mathrm{MF}$ species of Bangladesh, only 108 species are collected and preserved at the BAUBG. The remaining species would be collected and conserved. The conserved MF species might be multiplied and distributed among the rural people and different habitat restoration programmes. The development of improved cultivars and new value-added products and/or byproducts, and the expansion of the planting area of these MF species hold great potentials for improving nutrition and food security in Bangladesh and the world as well.

\section{ACKNOWLEDGEMENTS}

(Late) Prof. Dr M. Arshad Ali, the founder Curator, Prof. M. Mustafizur Rahman and the Present and previous Curators who have enriched the collection and curated the plant genetic resources of this Botanical Garden are thankfully acknowledged.

\section{REFERENCES :}

Ahmed, Z.U., Begum, Z.N.T., Hassan, M.A., Khondker, M., Kabir, S.M.H., Ahmad, M., Ahmed, A.T.A., Rahman, A.K.A. and Haque, E.U. (eds.) 2008a. Encyclopedia of Flora and Fauna of Bangladesh, Vol. 6. Angiosperms: Dicotyledons (Acanthaceae-Asteraceae). Asiatic Society of Bangladesh, Dhaka. pp. 1-408.

Ahmed, Z.U., Hassan, M.A., Begum, Z.N.T., Khondker, M., Kabir, S.M.H., Ahmad, M., Ahmed, A.T.A., Rahman, M.A.K. and Haque, E.U. (eds.) 2008b. Encyclopedia of Flora and Fauna of Bangladesh, Vol. 12. Angiosperms: Monocotyledons

(OrchidaceaeZingiberaceae). Asiatic Society of Bangladesh, Dhaka. pp. 1-552.

Ahmed, Z.U., Hassan, M.A., Begum, Z.N.T., Khondker, M., Kabir, S.M.H., Ahmad, M. and Ahmed, A.T.A. (eds.) 2009a. Encyclopedia of Flora and Fauna of Bangladesh, Vol. 7. Angiosperms: Dicotyledons (BalsaminaceaeEuphorbiaceae). Asiatic Society of Bangladesh, Dhaka. pp. 1-546. 


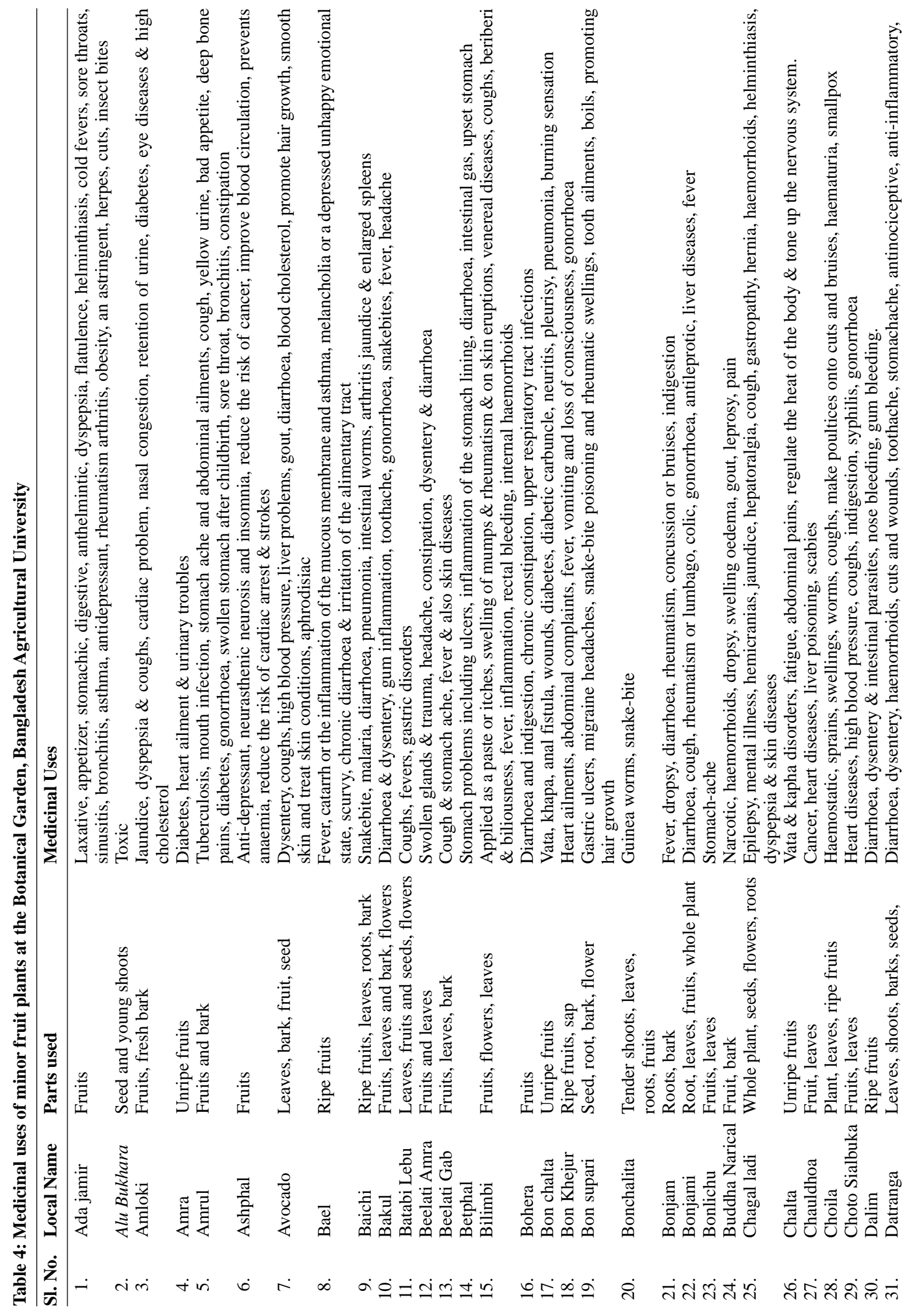


Ashrafuzzaman et al.

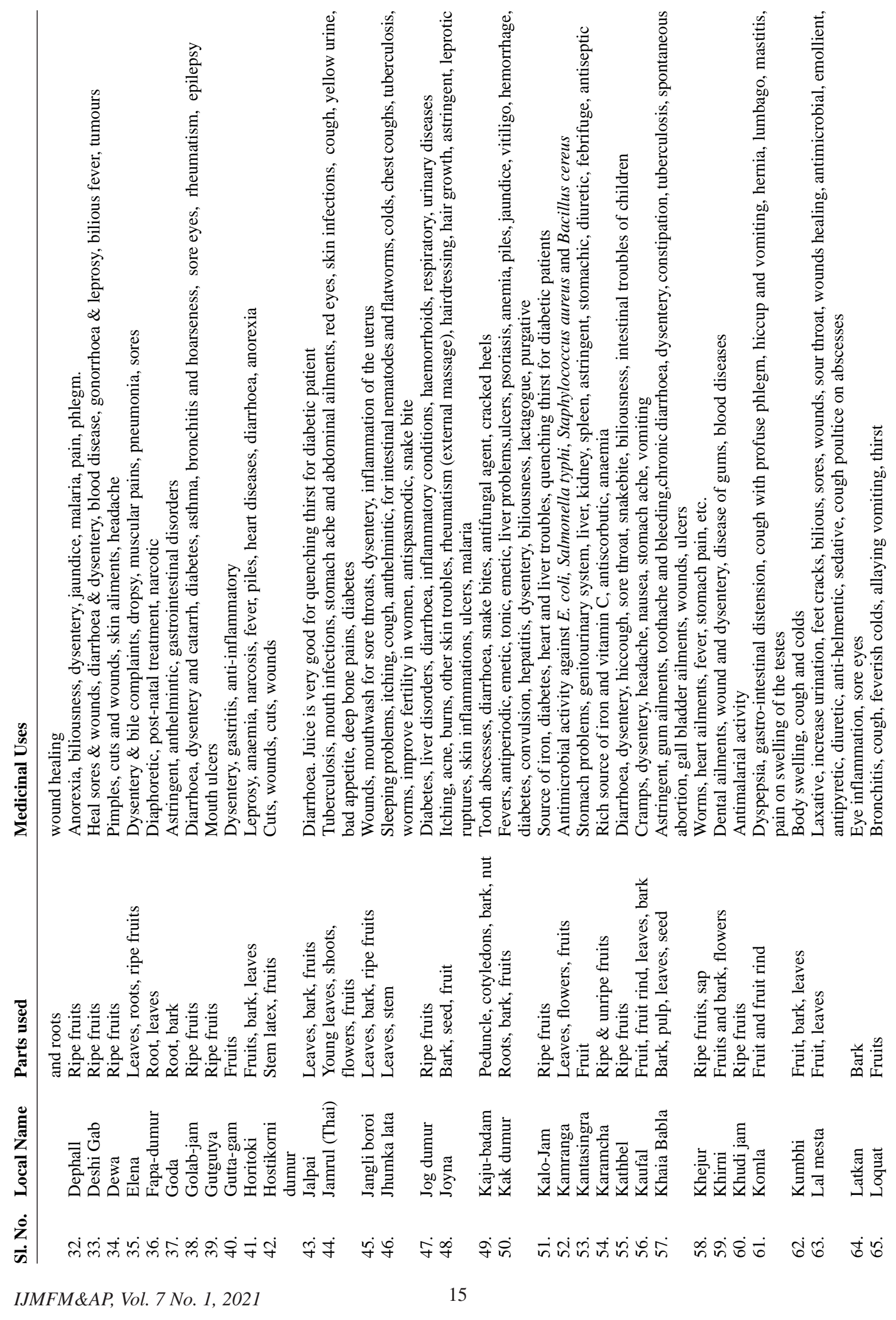


Conservation of minor fruit genetic resources at BAU

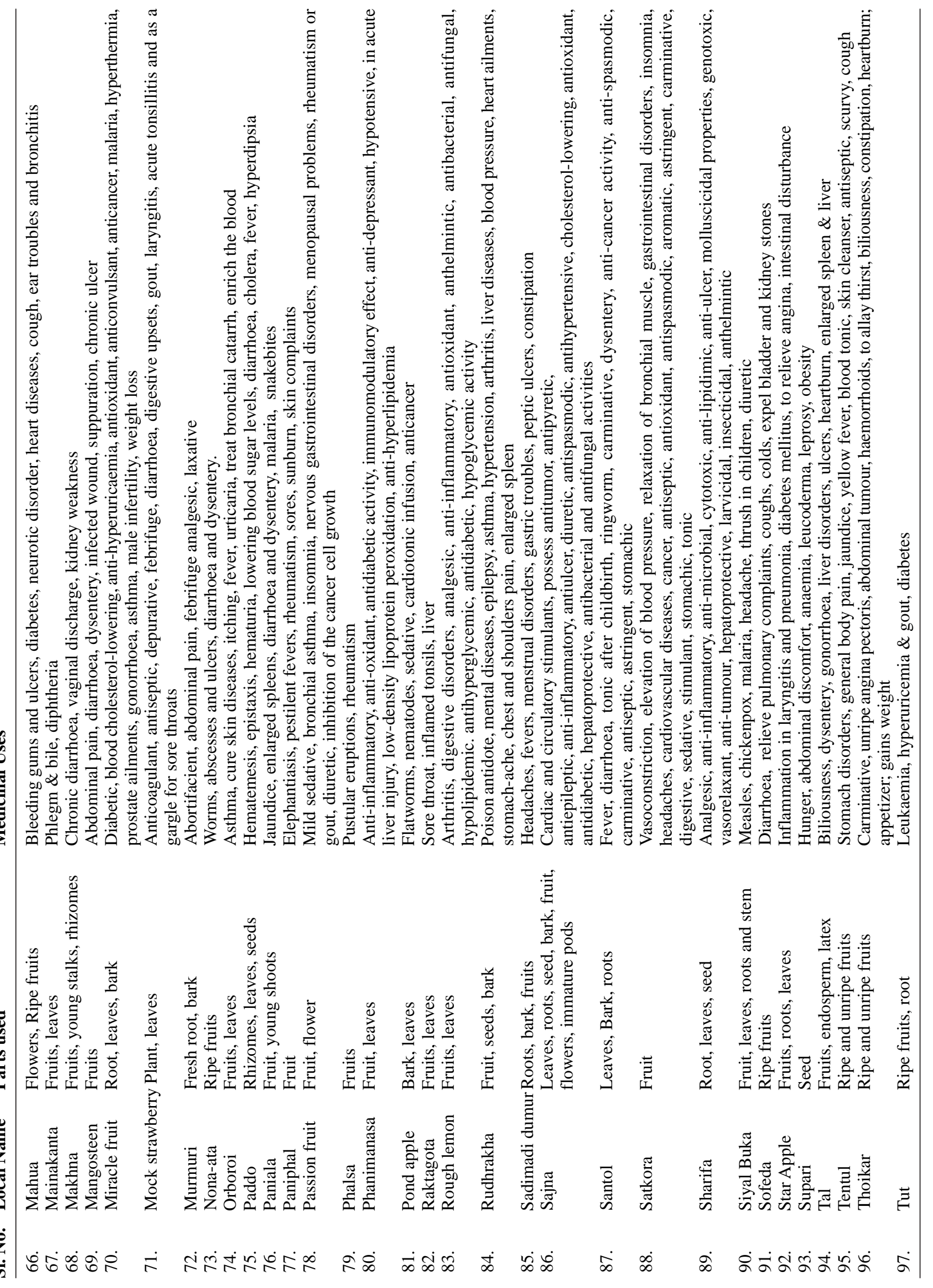




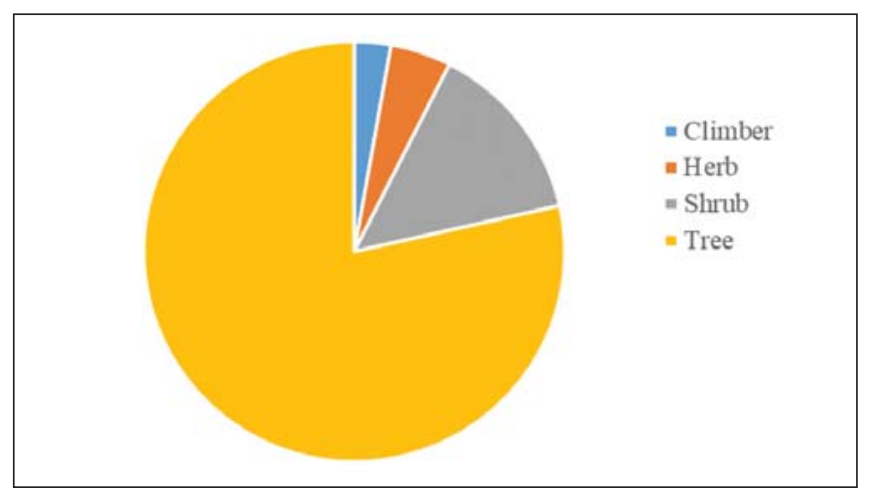

Fig. 7: Growth habit of minor fruits at Botanical Garden, Bangladesh Agricultural University

Ahmed, Z.U., Hassan, M.A., Begum, Z.N.T., Khondker, M., Kabir, S.M.H., Ahmad, M. and Ahmed, A.T.A. (eds.) 2009b. Encyclopedia of Flora and Fauna of Bangladesh, Vol. 8. Angiosperms: Dicotyledons (FabaceaeLythraceae). Asiatic Society of Bangladesh, Dhaka. pp. 1-474.

Ahmed, Z.U., Hassan, M.A., Begum, Z.N.T., Khondker, M., Kabir, S.M.H., Ahmad, M. and Ahmed, A.T.A. (eds.) 2009c. Encyclopedia of Flora and Fauna of Bangladesh, Vol. 9. Angiosperms: Dicotyledons (MagnoliaceaePunicaceae). Asiatic Society of Bangladesh, Dhaka. pp. 1-488.

Ahmed, Z.U., Hassan, M.A., Begum, Z.N.T., Khondker, M., Kabir, S.M.H., Ahmad, M. and Ahmed, A.T.A. (eds.) 2009d. Encyclopedia of Flora and Fauna of Bangladesh, Vol. 10. Angiosperms: Dicotyledons (RanunculaceaeZygophyllaceae). Asiatic Society of Bangladesh, Dhaka. pp. 1-580.

Altendorf, S. 2018. Minor tropical fruits - Main streaming a niche market. Food Outlook July 2018. FAO. pp. 67-75.

Ara, H., Khan, B. and Uddin, S.N. (eds.) 2013. Red Data Book of Vascular Plants of Bangladesh, Vol. 2. Bangladesh National Herbarium, Dhaka. pp. 1-280.

Dandin, S.B. and Kumar, N.K.K. 2016. Neglected and underutilized fruit species - an insurance against global mal and under nutrition. Regional Expert Consul., 3-5 December 2016, FAO Regional Office, Bangkok.

DoE (Department of Environment). 2015. Fifth National Report to the Convention on Biological Diversity. Min. Environ. \& Forest, Govt. of Bangladesh, Dhaka, pp. 1-164.
French, B. 2019. Food Plants International database of edible plants of the world, a free resource for all. In: Ravindran C. et al. (eds.) Proc. III Int. Symp. Underutilized Plant Species. Acta Hortic., 1241: 1-6.

Ghosh, S.N. (ed.) 2017. Minor Fruits: Nutraceutical Importance and Cultivation (3 Parts). Jaya Pub. House, Delhi.

Khan, M.S., Rahman, M.M. and Ali, M.A. (eds.) 2001. Red Data Book of Vascular Plants of Bangladesh. Bangladesh National Herbarium, Dhaka. pp. 1-140.

Krishna, H., Saroj, P.L., Maheshwari, S.K., Singh, R.S., Meena, R.K., Chandra, R. and Parashar, A. 2019. Underutilized fruits of arid \& semiarid regions for nutritional and livelihood security. Int. J. Minor Fruits Med. Aroma. Plants, 5(2): 1-14.

Pasha, M.K. and Uddin, S.B. 2019. Minor edible fruits of Bangladesh. Bangladesh J. Plant Taxon., 26: 299-313.

Rahim, M.A., Alam, A.K.M.A., Alam, M.S. and Anwar, M.M. (eds.) 2011. Underutilized Fruits in Bangladesh. Bangladesh Agriculture University, Mymensingh, pp. 1205.

Roya, A. and Bauri, F.K. 2019. Scope of minor fruit production in India. In: Ravindran C. et al. (eds.) Proc. III Int. Symp. Underutilized Plant Species. Acta Hortic., 1241: 43-49.

Sarwar, A.K.M. Golam. 2019. Plant Biodiversity and Conservation of Higher Plants in Bangladesh: Present Status and Future Prospects. In: Sangeetha, J., Thangadurai, D., Goh, H.C. and Islam, S. (eds.) Biodiversity and Conservation: Characterization and 
Utilization of Plants, Microbes and Natural Resources for Sustainable Development and Ecosystem Management. AAP/CRC, Florida, USA. pp. 259-288.

Sarwar, A.K.M. Golam. 2020. Medicinal and aromatic plant genetic resources of Bangladesh and their conservation at the Botanical Garden, Bangladesh Agricultural University. Int. J. Minor Fruits Med. Aroma. Plants, 6(2): 13-19.

Saúco, V.G. 2008. Global overview of underutilized tropical and subtropical fruits. In: Oh, D.-G. and Kubota C. (eds.) Proc. XXVII IHC Cultiv. Utiliz. Asian, Sub-Trop. Underutilized Hort. Crops. Acta Hort., 770: 77-85.

Siddiqui, K.U., Islam, M.A., Ahmed, Z.U., Begum, Z.N.T., Hassan, M.A., Khondker, M., Rahman, M. M., Kabir, S.M.H., Ahmad, M., Ahmed, A.T.A., Rahman, A.K.A. and Haque, E.U. (eds.) 2007. Encyclopedia of Flora and Fauna of Bangladesh, Vol. 11. Angiosperms: Monocotyledons (Agavaceae-Najadaceae). Asiatic Society of Bangladesh, Dhaka. pp. 1-399.
Srivastava, A., Bishnoi, S.K. and Sarkar, P.K. 2017. Value Addition in Minor Fruits of Eastern India: An Opportunity to Generate Rural Employment. In: Dutta, A.K. and Mondal, B. (eds.) Fruits for Livelihood: Production Technology and Management Practices. Agrobios (India), Jodhpur, India, pp. 395417.

Uddin, M.S., Uddin, S.B. and Lee, S.W. 2016. Ethnomedicinal Plants of Bangladesh. International Biological Material Research Center (IBMRC), Korea Research Institute of Bioscience and Biotechnology (KRIBB), Daejeon, Republic of Korea. 204 pp.

Uddin, S.N. 2006. Traditional Uses of Ethnomedicinal Plants of the Chittagong Hill Tracts. Rahman, M.M. (ed.), Bangladesh National Herbarium, Mirpur, Dhaka.

Yusuf, M., Begum, J., Hoque, M.N. and Chowdhury, J.U. 2009. Medicinal Plants of Bangladesh. $2^{\text {nd }}$ Rev. Enlarg. ed., Bangladesh Council of Scientific and Industrial Research Lab, Chittagong. 340 pp. 